This is an Accepted Manuscript of an article published by Taylor \& Francis in Journal of Marketing Management on 07/06/17 available online:

http://www.tandfonline.com/doi/full/10.1080/0267257X.2017.1328457 


\section{Towards an enhanced understanding of the behavioural phenomenon of multiple media use.}

\section{Abstract}

The central focus of this paper is the behaviour of individuals' when multitasking with media, wherein attitudes towards multiple media use and the management strategies employed when media multitasking are examined. A qualitative design is chosen for this in-depth study, using thirty four face to face interviews in the U.K., Germany and Australia. Findings confirm multiple media use as a central feature of everyday life, with a switching method of media multitasking widely acknowledged across all three samples. Broad similarities in attitudes, including the perceived costs and benefits of multitasking with media are uncovered across the samples, where media hierarchies and media synergies are generated alongside self-imposed restrictions to develop effective personal 'media multitasking portfolios'.

Keywords: IMC; media planning; multiple media use; media multitasking.

\section{Contribution}

This paper provides a unique insight into the complexities of the behavioural phenomenon of multiple media use, revealing: intricate behaviours; individual perceptions and personal management strategies among respondents in three countries, substantially contributing to this emerging research domain. The detection of bespoke 'media multitasking portfolios' is notable in relation to Integrated Marketing Communications (IMC). Moreover, this enhanced understanding is valuable to practitioners, in the comprehension of consumers' media multitasking behaviour, to plan efficient and effective IMC campaigns on behalf of clients. 


\section{Introduction}

Integrated marketing communications (IMC) is considered an important underpinning concept by the academic community, where it continues to be a topic of extensive debate within the literature (for example, Kitchen \& Schultz, 1998; Kliatchko, 2008; Laurie \& Mortimer, 2011). In the rapidly evolving and highly competitive marketing communications and advertising environment, practitioners strive to keep pace with the diverse range of channels of communication on behalf of their clients. A crucial element of the campaign planning process, for consideration by personnel in marketing communications and advertising agencies involved with IMC, is the selection of appropriate media channels to correspond with the media usage characteristics of target audiences (Keller, 2001; Fill \& Turnbull, 2016).

For individual consumers deciding which media to read, watch or listen to; the options are numerous, including traditional media (for example, television; press; radio; cinema) and newer internet based alternatives. For example, an individual choosing to read Cosmopolitan magazine can access the content by means of a paper copy; or electronically through a smartphone, tablet or laptop. Furthermore, user-generated content via web blogs and social media such as Instagram, YouTube and Facebook, extend the range of media options. As a consequence, individuals' now enjoy considerably more control over their media consumption (Pilotta \& Schultz, 2005). The evolution of on-demand media services offered by providers such as Sky (in the television medium), allows additional media control (Webster \& Ksiazek, 2012). The variety afforded by the contemporary media environment, in conjunction with continual technological advances, offers the ability for individuals' to engage in multiple media use. The behavioural phenomenon of multiple media use is established in the literature (for example, Pilotta \& Shultz, 2005; Foehr, 2006; Jeong \& Fishbein, 2007; Bardhi, Rohm \& Sultan, 2010; Brasel \& Gips, 2011; Kononova \& Chiang, 2015). Moreover, other empirical studies indicate that multiple media usage occurs in a variety of locations; 
with the home, restaurants or bars, and the workplace acknowledged as settings in which multiple media use occurs (for example, Enoch \& Johnson, 2010).

Continual advancements in media channel availability present opportunities and challenges for all participants of IMC: marketing communications, creative and media planners, working on behalf of their clients; media owners providing and selling airtime and space; and media consumers. While an extensive range of choice is enjoyed by consumers of media; this presents a complex set of selection decisions for a media planner, working on a client's behalf to maximise the attention of a specific target audience for the marketing communications and advertising activity of their brand. A central tenet of the media planning function is; the matching of relevant media to a specified target audience effectively and efficiently, to gain maximum exposure at minimum cost on behalf of clients (Danaher, 2007). The benefits of media synergy, allied with the concept of IMC, suggest that a combination of media can prove more effective than a single channel media campaign (Laurie \& Mortimer, 2011; Kliatchko, 2008). With this in mind, it is argued that although media planning for a multiple media usage scenario creates increased complexity at the planning stage, synergistic benefits are conceivable (Bardhi et al., 2010; Voorveld, 2011). Hence, for the marketing communications practitioner, an enhanced understanding of this behavioural phenomenon is advantageous.

In the emergent research domain of multiple media use (Lin, 2009); extant studies primarily comprise descriptive work, revealing various combinations of multiple media use by consumers; although recent empirical work addresses the antecedents and outcomes of media multitasking (for example, Carrier et al., 2009; Jeong \& Fishbein, 2007; Kononova \& Chiang, 2015; Srivastava, Nakazawa \& Chen, 2016). However, with the exception of a study by Bardhi et al. (2010), the underlying attitudes towards consumers' use of multiple media and the ways in which individuals' manage their multiple media use are not examined in depth. Hence, there remain gaps in understanding of the complexities of this behavioural phenomenon. Accordingly, this paper details a 
study which updates and extends the scope of empirical work in this domain into the U.K., Germany and Australia; substantially contributing to our understanding of individuals' approaches to multiple media use. The aim of this study is to explore the intricacies of the media multitasking behaviours of individuals and their underlying attitudes towards multiple media use among Digital Natives in the U.K., Germany and Australia; thus enabling comparisons between individuals in these countries and those in the previous U.S. study by Bardhi et al. (2010).

\section{Literature review}

The literature reveals an emergent body of empirical work examining the topic of multitasking in the media context. Extant empirical work in this domain includes the investigation of: the generational composition of media multitasking individuals; the occurrence of media multitasking; media multitasking combinations; and the frequency of switching behaviour. Preliminary exploration of individuals' perceptions of media multitasking behaviour and an evolving consideration of the antecedents and consequences of multiple media use is also evident in more recent studies.

Multitasking is confirmed as a behavioural concept; to perform 'multiple task goals in the same general time period by engaging in frequent switches between individual tasks' (Delbridge, 2000). Furthermore, Rosen et al. (2013) distinguish multiple media use as a special case of multitasking. A review of extant definitions of the phenomenon of multiple media use reveals a variation in labelling: 'media multitasking' (Foehr, 2006; Bardhi et al., 2010; Wang et al., 2012); 'simultaneous media usage' (Pilotta et al., 2004; Pilotta \& Shultz, 2005) and 'multitasking with media' (Jeong \& Fishbein, 2007). Discrepancies are apparent concerning: commercial and non-commercial media; media and non-media activity; multiple media usage, at a single point in time or during a given period. The definition offered by Delbridge (2000) is adapted for the media context of this study; conceptualising multiple media use as 'engaging in multiple media tasks in the same general time period by engaging in frequent switches between individual media tasks'. For example; watching a 
television programme, searching the Internet and replying to a text. In this scenario, media activities may occur on one, two or three devices.

Multiple media users are examined in empirical work by Carrier et al. (2009) among three generations: 'Baby Boomers' (born between 1946 -1964), 'Generation X' (born between 1965 -1979) and the 'Net Generation' (born between 1980 - present). Findings confirm that media multitasking is dominant among the 'Net Generation', followed by 'Generation X' and the 'Baby Boomers'. Other studies affirm this finding (for example, Foehr, 2006; Pilotta \& Shultz, 2005; Carrier, Rosen, Cheever \& Lim, 2015) in accordance with expectations; as the 'Net Generation' or 'Digital Natives' have grown up through three decades of extraordinary progress in the development of media technology. Digital Natives (born after 1980) are classified as 'all native speakers of the digital language of computers, video games and the Internet' (Prensky, 2001 p.1).

Pilotta and Shultz (2005) reveal that $40-65 \%$ of total media consumption time comprises multiple media activity. Heavy media consumption is an indicator of the likelihood of individuals' to multitask (Pilotta et al., 2004; Foehr, 2006). In their qualitative study, Bardhi et al. (2010, p.328) conclude that 'media multitasking is the way young consumers interact with commercial media'. While such qualitative findings are not generalisable to a general population, this finding concurs with Pilotta et al. (2004) and Pilotta and Shultz (2005), confirming the practice of multiple media use. Pilotta and Schulz (2005) and Pilotta et al. (2004) examine SIMM data (BIGresearch), identifying an assortment of media multitasking combinations. From this study and further work by Carrier et al. (2015), it is evident that there are several prevalent media combinations including: TV and Internet; TV and newspapers; email and text; instant messaging and music. Moreover, during a period of multiple media use, more attention is given to one medium; the foreground medium, than the other; the background medium (Pilotta \& Schultz, 2005). Similarly, Brasel and Gips (2011) observational work, using a media combination of computer and television, supports this conclusion. Notable findings 
are exposed by Brasel and Gips (2011) who report that the speed of switching between TV and computer is found to be extremely fast and frequent, measuring an average of four switches per minute. In a separate single device study, this finding is affirmed by Yeykelis, Cummings and Reeves (2014), who recorded switches between media content on personal computers every 19 seconds during a one day period. Although providing important information on the behavioural aspects of multiple media use, extant studies predominantly restrict their analysis to two-way combinations of media. However, it is apparent from viewing individuals' everyday media use, that it often includes combinations larger than two.

Recent literature reveals an evolving body of work investigating the antecedents of multiple media use; in which individual media ownership, media availability and an array of audience related features are discussed. Individuals' media ownership, represented by owned devices (for example; smartphones; tablets; laptops; televisions; radios) in conjunction with the extent of individuals' available access to media (for example, having access to a television in the bedroom) are identified as pre-requisites to multiple media use (Jeong \& Fishbein, 2007; Wang \& Tchernev, 2012; Kononova \& Chiang, 2015). Age is exposed as the chief audience feature, as discussed earlier, with younger audiences more likely to participate in multiple media use than older ones (for example, Carrier et al., 2009; 2015; Jeong \& Fishbein, 2007; Duff, Yoon, Wang \& Anghelcev, 2014; Srivastava et al., 2016). Gender has also been found to differentiate media multitasking (Jeong \& Fishbein, 2007; Duff et al., 2014), with females more likely to multitask with media than males, but this finding is not widely supported. Individual differences, such as creativity (Duff et al., 2014) and the propensity for sensation seeking (Jeong \& Fishbein, 2007; Duff et al, 2014) are also uncovered as predictors of multitasking in the media context. Motivations to multitask with media include features such as: a need for simplicity; personal control; efficiency; information; connection and entertainment (for example, Duff et al., 2014; Kononova \& Chiang, 2015; Hwang, Kim \& Jeong, 2014; Srivastava et al., 2016). Furthermore, the behavioural character of media multitasking is predicated by individuals' preference for such activities (Srivastava et al., 2016), termed polychronicity (Kononova \& Chiang, 
2015). Consequences of multiple media use are also addressed in this growing research domain, indicating intrinsic advantages and disadvantages of multiple media use across a range of media pairings (for example, Wang \& Tchernev, 2012; Ophir, Nass \& Wagner, 2009; Voorveldt, 2011; Srivastava, 2013). While extant empirical work makes a valuable contribution to knowledge in this domain, there remains a requirement for further depth of understanding of the underlying attitudes of individual consumers in relation to their media multitasking behaviour.

An exploratory study by Bardhi et al. (2010) instigates the in-depth examination of multiple media use, with reference to the Motivation, Ability and Opportunity (MAO) model, which forms antecedent conditions of the Elaboration Likelihood Model ELM (Petty \& Caccioppo, 1986). The ELM contends that the likelihood of elaboration of advertising messages is higher when individuals possess the motivation, ability and opportunity to process them. Bardhi et al. (2010) argue that while inquiry in cognitive psychology may suggest that individual attention is reduced by media multitasking behaviour (thus reducing MAO), it is possible that the influence on MAO may be positive if individuals develop effective coping strategies for their multiple media use. Themes emerging from this qualitative study illustrate the paradoxical nature of the practice of media multitasking, highlighting several perceived benefits (efficiency; assimilation; control; engagement) and costs (inefficiency; chaos; disengagement; enslavement). Additionally, four distinct coping strategies for consumers' management of multiple media are identified.

In summary, multiple media use is established as an emerging research domain within the literature (Lin, 2009). Empirical work to date confirms the behavioural phenomenon of multiple media use, identifying assorted 'media with media' and 'media with other activity' combinations, in which frequent switching behaviour is confirmed in single and multiple device media multitasking. Early empirical studies are predominantly descriptive in nature and are constrained by their analysis of only two-way combinations of media multitasking. However, an evolving body of research examines the antecedents of media multitasking, advancing knowledge of the media ownership and audience 
factors which precede this behaviour. Nevertheless, the underlying attitudes towards multiple media use are not yet fully addressed; although the qualitative study by Bardhi et al., (2010) initiates this aspect of inquiry. Following this work, there is scope to deepen the investigation of the attitudes of individuals' towards their multiple media use. Moreover, there is a gap in understanding of the intricacies of individuals' behaviour when multitasking with media, indicating a requirement for further empirical work to develop greater insight into this behavioural phenomenon. A comprehensive understanding of consumers' multiple media use behaviour is essential for practitioners in marketing communications and advertising media planning, seeking to optimise their clients' media budgets through multi-media IMC campaigns.

\section{Aim of the study and research methods}

Our study updates and extends the scope of previous work on the investigation of multiple media use, to deepen our understanding of the phenomenon. The aim of this study, a partial replication and extension of the work of Bardhi et al. (2010) in the U.S., is to explore media multitasking behaviours and underlying attitudes towards multiple media use among Digital Natives. The study is conducted in the U.K., Germany and Australia, since differences in media consumption patterns and availability of media channels between these countries are evident (Reuters, 2012). Digital Natives (born since 1980, Prensky (2001)) are chosen for this study, as media multitasking is most prevalent amongst this group (Carrier et al., 2009; 2015).

Consistently, our research objectives are; among Digital Natives in the U.K., Germany and Australia:

(a) To explore multiple media use behaviours;

(b) To explore the perceived benefits and costs of media multitasking;

(c) To understand the strategies employed when multitasking with media

Objective (a) establishes types of behaviour, providing the background for objective (b), which examines perceptions of media multitasking; while objective (c) focusses on the strategies employed by individuals' when they engage in multiple media use. 
A qualitative research design is considered appropriate for this exploratory study, intended to gain an in-depth understanding of media multitasking behaviour (Mariampolski, 2001) in this emerging research domain (Lin, 2009). Individual interviews are used, to allow respondents to articulate their ideas, attitudes and reasons for multiple media use without being intimidated or influenced by others (Malhotra and Birks, 2007). Semi-structured interviews are considered most suitable, as they facilitate comparison whilst allowing interesting points to be developed (Savin-Baden \& Howell Major, 2013). A convenience approach, using non-probability quota sampling, was employed for selection of respondents. Subsequently, semi-structured, face to face, in-depth interviews were conducted among Digital Natives aged between 18-30 (Prensky, 2001), who claimed to multitask with media. Twelve interviews were conducted in the U.K. and Germany, with ten in Australia (each with an equal number of male and female respondents); at which point there was a reasonable expectation that no new insights would have been gained from conducting further interviews, hence achieving theoretical saturation (Bryman \& Bell, 2011; Richie \& Lewis, 2003). The interviews were conducted in jointly agreed settings, lasting approximately one hour. All interviews were audio tape recorded and transcribed. In Germany, the interviews were conducted in German, with transcriptions translated into English prior to analysis. The data analysis procedure advocated by Miles and Huberman (1994) was adopted, comprising: data reduction; data display and verification; in which the coding method used open, axial and selective codes. A manual coding procedure was utilised in the analysis of all interview transcriptions, aided by Computer Assisted Qualitative Data Analysis Software (CAQDAS), specifically NVivo10. The use of CAQDAS assisted in the identification of key themes and in the management of data from thirty four interviews (Rettie, Robinson \& Radke, 2008). The utilisation of a three country sample ensured triangulation (Denzin and Lincoln, 2011).

Yardley (2000) recommends four quality criteria to be observed by qualitative researchers, which are applied in this research study. Sensitivity to context is established by referring to extant academic literature and contemporary evidence concerning the media environment in which multiple media 
occurs. A systematic process was adopted for data collection and analysis of findings as specified in preceding sections, hence demonstrating commitment and rigour. During the research process, techniques are plainly articulated and explained, with comprehensive notes maintained at all stages of the study (for example, all interview transcriptions are stored for future reference), thus ensuring transparency and coherence. Furthermore, impact and importance criteria are met, as the study is situated in the background of extant empirical work on the topic of multiple media use. Lastly, the findings of the study and its implications for academics and practitioners are elaborated in the later discussion, together with proposals for future research directions in this domain.

\section{Findings}

The study revealed that in the U.K., Germany and Australia, respondents described their media multitasking behaviour as a notable activity within their daily routines of researching information, entertaining themselves and communicating with friends and family. Furthermore, despite reported differences in media availability and patterns of media consumption in the three sample countries (Reuters, 2012); the findings of this qualitative study uncover broadly similar themes among respondents in the U.K., Germany and Australia in relation to their multiple media use. In the following sections, the behaviours surrounding multiple media use are discussed; followed by an examination and comparison of the perceived costs and benefits of media multitasking by the respondents in different countries. Lastly, the strategies employed by media multitasking individuals are explored. While the similarities and differences between the U.K., Germany and Australia are not the subject of detailed scrutiny in this paper; these are demonstrated through the verbatim quotations from the U.K., German and Australian respondents in succeeding sections, where subtleties are evident.

\section{Multiple media use behaviours}

Across the three sample groups in the U.K., Germany and Australia, respondents reported that media multitasking is a central part of everyday life. Moreover, the concept of a switching method of 
media multitasking is widely acknowledged by respondents in all three samples. The findings provide confirmation of temporary pauses when respondents are multitasking, to allow more effective management of multiple media; for example, pausing a programme on the television to attend to a social media alert or text message. The discovery of the approaches to switching between different media is of specific interest, showing that individuals' decisively suspend the use of one medium in preference of another (for a period of time); and then return to that medium as shown in the following quotes:

I actually multitask with media most of the time. I think I use one medium only when I watch news in the morning and when I cook and watch TV at the same time. But otherwise it's always the case that I multitask with media.' (Germany: 12)

'If the TV's on and I'm not actually watching anything, like if it's...something that I don't need to concentrate on, I'll have my iPad on, my laptop on, my extra screen on and I'll be doing things and if it catches my attention I'll watch it.' (Australia: 5)

'Your main focus is on whatever you are doing. It's not a 50:50 split or a 60:40 split. I'd say it's mainly one or the other.' (U.K.: 11)

Switches in attention are found to have several causes; the most frequently cited being an interruption from one of the media or a break in the content, such as for advertisements. While multitasking with two media was common (for example, laptop or tablet and TV; phone and TV), several respondents described situations when they used three or more media concurrently (for example; smartphone, laptop or tablet and TV). A variety of different combinations of media were described by our respondents, and evidence was found of multitasking both with separate media and multitasking within a single device or medium, which depends on the location of the respondent. Single device multitasking is a notable finding of this study, confirming previous empirical work (Brasel and Gips, 2011; Yeykelis et al., 2014). Examples of switching scenarios are illustrated in the following quotes:

'I'll put my phone down and concentrate on it (television) until the adverts are on or something, then I'll pick up my phone.' (U.K.: 7)

'Often times I'll read an article for a bit and then I'll go onto Facebook and then watch a video for a bit and then I'll go to the news...so I'll chop and change' (Australia: 10). 
'When I'm at home, I multitask with media. Then the TV is switched on, I use Facebook, read emails and check my mobile phone. But when I'm on the go, I only check my mobile phone every now and again and nothing else.' (Germany: 7)

Whilst the media are often used independently, indications were found of linked or synchronised media usage; for example, using the Internet to obtain further information on a news story or viewing tweets or exchanging private messages from friends relating to a live television programme. Synchronised media multitasking is illustrated in these quotes which portray a variety of situations in which this behaviour occurs:

'One will be prompted by the other, you'll be watching TV and then because you see something on TV you'll Google it. Or, if you're listening to something and you'll think, I wonder what that band's doing now or something like that...you'll Google it.' (U.K.: 11)

'I mostly watch things which are more fun to watch together. I think that this, by the way, is also the reason for swapping opinions with others via my iPhone when I watch TV alone... because I am in contact with my friends at the same time I feel less alone.' (Germany: 8)

'I'll take my radio (to the football game) because I find it more interesting to watch the game when you can hear who all the players are...so it keeps you updated as to what is happening and then I would use my phone to check who's kicked the most goals and that sort of thing.' (Australia: 2)

However, some exclusive media events are evident, most notably cinema, which is rarely combined with any other media. In a similar way, a pre-planned film on television is often watched without interruption. Additionally, reading a weekend newspaper (or a book) is also considered a solitary media activity by some, as indicated by the following quotes:

'Cinema is more of an event, so everything goes off.' (U.K.: 9)

'I only use the TV on its own when I have consciously picked a movie I really want to watch. And then I don't use any other medium at the same time...if I really decide to watch something, I focus on it and I don't like to be interrupted then.' (Germany: 12)

'Because the (weekend) newspaper is relaxing to me, I think it winds down my screen usage...so trying just to give myself a little time and space from emails and the daily kind of routine Monday to Friday.' (Australia: 3)

\section{Benefits of media multitasking}

The perceived benefits of media multitasking, reported by respondents in the three country samples are displayed in Table 1, alongside the findings from Bardhi et al. (2010). From inspection of Table 1, 
it is immediately clear that there are similarities across all three countries in the efficiency and control benefits cited by respondents in this study, in accordance with Bardhi et al. (2010). Similarly, assimilation (not mentioned by the German and Australian samples) is considered a benefit of multiple media use in the U.K. sample, again supporting Bardhi et al. (2010). Additional findings of this study include the perceived benefits of emotional gratification and social engagement (Germany; Australia); relating to engagement benefits revealed by Bardhi et al. (2010). Furthermore, this study uncovers connectedness (Germany; Australia) and access to information (U.K; Germany; Australia) in relation to the perceived benefits of multiple media use. 
Table 1: Respondents' perceived 'benefits' of media multitasking

\begin{tabular}{|l|l|l|l|}
\hline Bardhi et al., U.S. (2010) & U.K. (2013) & Germany (2013) & Australia (2014) \\
\hline Efficiency & Efficiency & Efficiency & Efficiency \\
\hline Assimilation & $\begin{array}{l}\text { Assimilation (due to } \\
\text { time pressure) }\end{array}$ & & Control \\
\hline Control & Control & $\begin{array}{l}\text { Improved media experience } \\
\text { (includes control) }\end{array}$ & $\begin{array}{l}\text { Social (engagement) } \\
\text { (rather than engagement) }\end{array}$ \\
\hline Engagement & $\begin{array}{l}\text { Connectedness/keeping one } \\
\text { company }\end{array}$ & Connectedness \\
\hline & $\begin{array}{l}\text { Extended information } \\
\text { access }\end{array}$ & Access to information \\
\hline
\end{tabular}




\section{Efficiency}

An important theme revealed across all samples in this study, is that multiple media use is linked with feelings of efficiency by the respondents, in line with previous work by Bardhi et al. (2010). The need for efficiency by the respondents is strongly linked with time pressures, both internally and externally generated, whereby there is a feeling that to achieve all of their tasks, it is necessary to multitask with media. Media multitasking is linked with a reduction in the amount of time or effort to get things done; for example, gathering information from news media and social media to stay 'well informed' and 'up to date'. For some, there is also a perception that tasks can be finished more quickly when more than one medium is used at the same time. Furthermore, some respondents reported that listening to music on the radio facilitates work as it generates a 'good mood'. Illustrations of feelings of efficiency when media multitasking are found in the following quotes:

'Benefits include saving time and being able to do multiple things at the same time.' (Australia: 10)

'Music improves my concentration and I can work much better.' (Germany: 7)

'When I was studying politics it was kind of essential to keep up to date with all the current affairs, so for me it was a habit of reading the newspaper while listening to the news or Question Time/ Newsnight while reading something else.' (U.K.: 6)

\section{Assimilation}

Along a similar theme, in accordance with Bardhi et al. (2010), the ability to assimilate vast quantities of online content and user generated content is considered a benefit of media multitasking by U.K. respondents, who feel pressured to stay in touch with a variety of information sources: personal; entertainment and news based, on an ongoing basis, 'There is so much content out there, you can never get it all in a lifetime. So, in order to get as much as possible and get a good overview of what is going on you need to have multiple streams now.' (U.K.: 6)

\section{Control}

Supporting the findings of Bardhi et al. (2010), all respondents in the U.K., Germany and Australia discussed a sense of control in relation to their media multitasking behaviour, both in work and 
entertainment settings. Many respondents (particularly in the German sample) mentioned feelings of control while engaged in multiple media use, in association with an improved media consumption experience, as indicated by these quotes:

'If the television programme is boring, with media multitasking I feel I can control that I look at something more interesting on the iPad or my laptop...and I think I'm more involved in this content because I have consciously sought it out.' (Germany: 8)

'In a work situation I think it's good, I feel in control. I like media multitasking when I'm working because I actually need everything that's on.' (Australia: 5)

Control is also alluded to by many respondents in relation to their multiple media management strategies, discussed later.

\section{Engagement}

Engagement, one of the main benefits of media multitasking uncovered by Bardhi et al., (2010), also features in the responses of individuals in our German and Australian samples, mentioned in terms of emotional gratification and social engagement respectively. Among the German sample, media multitasking is felt to create; satisfaction, entertainment and a feeling of relaxation, thus supporting an individual's emotional wellbeing; 'If I sit in front of the TV, I don't need to concentrate on anything. I just use it to relax and as background noise. Then it's not important whether I'm working efficiently or not. In this case, it definitely makes me more satisfied if I can use multiple media simultaneously.' (Germany: 12). Similarly, among Australian respondents, there is a feeling that media multitasking in the presence of others can engender a social experience, as revealed by this quote:

'Even if we're all sitting together in the same room, we'll still be communicating on that Viber. Just for...sometimes just for fun, sometimes somebody in a different room, sometimes while everyone is in the same room. We still tend to use it while the TV is on in the background.' (Australia: 7)

\section{Connectedness}

A feeling of connectedness, uncovered in this study, was revealed among respondents in Germany and Australia, who cited the ability to be constantly available to friends and family as one of the 
main benefits of media multitasking. Other related benefits include the need for background noise and to combat loneliness (Germany), as illustrated by these quotes:

'It gives me peace of mind that people can get in touch with me any time.' (Germany: 3)

'Because I am time poor and I see a lot of what's going on in their life through these various media I don't feel quite so removed.' (Australia: 3 )

'Since I live on my own and it's often very quiet in my apartment, I use my TV in the background' (Germany: 4)

\section{Information access}

Across all three samples in this study, respondents felt that extended access to various sources of information and the additional information afforded by multiple media use is of benefit to them. Respondents talked about having additional benefits and supplementary information related to media content or being better informed by having an additional medium when engaged in multiple media use, as indicated in these quotes:

'Media multitasking provides me with an added value because I can look things up immediately that I would otherwise forget if I intend to do it later.' (Germany: 10)

'So, advantages would be having access to so many things, so many options, you've got research...there's just...you can learn such a lot from the comfort of your own home and your media channels' (Australia: 8).

\section{Costs of media multitasking}

Table 2 illustrates the perceived costs of media multitasking, reported by respondents in the three country samples, along with the findings of the Bardhi et al. (2010) study. Paradoxes are again evident, for example; efficiency and engagement, cited as benefits in Table 1, appear here as costs (inefficiency and disengagement). Similarities exist in the perceived costs of multiple media use, with inefficiency and enslavement (or habit) cited by all three samples, supporting the previous findings of Bardhi et al. (2010). A further perceived cost of media multitasking found in the U.K. and Australian samples is chaos, in agreement with the findings of Bardhi et al. (2010); while disengagement is a cited cost by all samples except Germany. In this study, additional costs described by U.K., German and Australian respondents include distraction, or an inability to focus on 
one thing whilst engaged in multiple media use. Correspondingly, a reduction in attention is expressed among German and Australian respondents when multitasking with media. 
Table 2: Respondents' perceived 'costs' of media multitasking

\begin{tabular}{|c|c|c|c|}
\hline Bardhi et al., U.S. (2010) & U.K. (2013) & Germany (2013) & Australia (2014) \\
\hline Inefficiency & Inefficiency & Inefficiency & Inefficiency \\
\hline Chaos & $\begin{array}{l}\text { Similar effect termed } \\
\text { 'over-stimulation' }\end{array}$ & & Chaos \\
\hline Disengagement & (Social) disengagement & & Anti- social \\
\hline \multirow[t]{3}{*}{ Enslavement } & $\begin{array}{l}\text { Habit (rather than } \\
\text { addiction/enslavement) }\end{array}$ & Enslavement & Enslavement \\
\hline & Distraction & $\begin{array}{l}\text { Inability to focus on } \\
\text { one thing }\end{array}$ & Distraction \\
\hline & & Reduced attention & Attention span suffers \\
\hline
\end{tabular}




\section{Inefficiency}

The perceived costs of media multitasking in all samples were found to be the distraction from a single task or activity and related inefficiencies, such as a lack of focus, 'I don't always pay full attention. When using two media at the same time, then it isn't possible to satisfy any of the things because both are done only half-heartedly' (Germany: 2). Predominantly this occurs when one media distracts the user, causing them to miss something deemed important. This is followed by a need to take action to correct it, leading to inefficiency. Individuals also reported that media multitasking can result in an inefficient use of their time. Specific examples of inefficiencies are revealed in the following quotes:

'I'll be watching the television and I have the computer, I am like, Internet shopping and I'm watching the show and I sometimes pause it, I don't want to miss something, or I'll rewind it back because, you know, Sky has that feature' (U.K.: 7)

'Let's say you're doing a spreadsheet or something quite complex, by the time you go to Facebook and read a post or post something and use your brain to figure out what you're actually going to write, you're still thinking about the spreadsheet at the same time you're thinking about the post. Then when you go back to your spreadsheet you need to just go back to what was your train of thought before you went on Facebook. So you're losing efficiency there for sure.' (Australia: 4)

Chaos

Chaos, over-stimulation or feeling overwhelmed by input, is reported by all samples (except

Germany) as a cost of media multitasking. One U.K. respondent described media consumers as 'having so much blasted at them', suggesting feelings of an uncontrollable experience. The findings of this study are in accordance with those of Bardhi et al. (2010) in this regard, as illustrated below:

'In some ways it can be negative, over stimulation, too much information, you can never find out all the facts, you can never get all the information about something, there is always something new going on.' (U.K.: 6)

'It's frustrating because, I'm losing focus. It's kind of hard; my mind is all over the place.' (Australia: 8)

\section{Disengagement}

Disengagement from media was found to be associated with multitasking by many respondents in the U.K. and Australia, consistent with Bardhi et al. (2010). In addition to the disengagement from 
media, the respondents in this study also felt that they became socially disengaged, feeling disconnected with others as a result of media multitasking. Some U.K. respondents reported that they felt unable to fully concentrate on media when multitasking, losing the ability to focus on one thing, which resulted in 'blanking out' or 'glossing over' certain elements of a medium, as reported in these two quotes:

'Multitasking, having multiple streams coming at you, if you listen to the TV and listen to the radio at the same time, what's actually going on in your head, were you paying attention at all? Are you just glossing over, getting used to it as background noise?' (U.K.: 6)

Concerns about social disengagement led to respondents limiting their media multitasking usage whilst in the company of other people, 'I think it's rude' (Australia: 5).

\section{Enslavement}

A feeling of enslavement or addiction to media multitasking, as described by Bardhi et al. (2010), was also encountered among the German and Australian respondents, whereas in the U.K. this was described as habitual behaviour, as described by this respondent:

'Like I said it's in our consciousness and when you're on the Internet you do find yourself logging in automatically, it's just scary how you're already putting in your password and you don't even realise 'hang on I'm on Facebook at the moment', it's incredible. So, yeah, the habit is really strong now, and I think the same with TV, just having a bit of background noise' (U.K.:10)

Enslavement is related to the addictive nature of media multitasking, where respondents feel an increasing desire to multitask and a dependency on media, as described in these two quotes:

'I constantly feel the urge that I want to look something up if I use media. It's hard to stop myself from doing it...It has become a compulsion, even a fear of missing something' (Germany: 8)

'I feel like I check it all the time...I think that might not be the great thing about it, it gets a bit obsessive' (Australia: 6)

\section{Distraction}

Distraction is considered a cost of multiple media use by all three samples in the study, occurring when one media distracts an individual, causing them to miss something deemed important in another medium and resulting in a need to take some sort of action; for example, re-watching the 
missing footage. In addition, frequent multiple media use is considered to result in a loss of focus on a single activity or task. The following quotes underline this perception:

'If you are flipping between the two, you are probably not getting the most out of one or the other; you are just getting bits of both.' (U.K.: 3)

'When I use a TV and a laptop at the same time, which I do quite often, I think that's a bit extreme. It shows that I feel bored very easily and, what is worse, that I can't focus on one thing anymore.' (Germany: 7)

'I don't like listening to music and watching TV at once, So... like music and TV, I just find that really distracting.' (Australia: 7)

\section{Reduced attention}

Respondents in Germany and Australia reported a reduction in their attention levels or attention span, considering this to be a result of their continuing excessive multiple media use. In turn, this is believed to lead to reduced recognition and recall of media content, due to the division of attention between the different media, as shown by the following quotes:

'When I watch a movie and read an email or a tweet at the same time, of course I catch less of the movie. But what I read gets $100 \%$ of my attention. It's switching between the media all the time. When looking back to the TV, then I'll catch 100\% of the TV again. My attention is either on one thing or another. But then it's also harder to get back into the story again.' (Germany: 3)

'Since I got a smart phone, I feel like my media access has been more sporadic and I feel like my attention span maybe suffers slightly because of that.' (Australia: 1)

\section{Management strategies}

Consistent with initial findings by Bardhi et al. (2010) that consumers developed coping strategies for their media multitasking activities, three distinct strategies are identified among our U.K., German and Australian samples. In the management of their media multitasking activities, respondents: create media hierarchies; develop media synergies and use self-imposed restrictions.

\section{Creation of media hierarchies}

By creating media hierarchies, users control how intensively they interact with various media when multitasking. In this scenario, some media are consumed as foreground and others as background, confirming previous work (Pilotta \& Schultz, 2005; Brasel \& Gips,2011). Traditional media such as 
television and radio are often used as background, while new media are regularly consumed in the foreground. For example, a television set may be used as a background device, whilst a laptop, tablet or phone is in the foreground. Many respondents did not feel that television was engaging enough to be watched on its own, 'It's so boring to only watch TV. It doesn't challenge me enough. Maybe it's just the nature of traditional media.' (Germany: 10). Correspondingly, television programmes are often watched 'on demand', allowing them to be paused, hence aiding the multiple media user. Examples of such media hierarchies are illustrated in the following quotes:

'So, sometimes I like to have TV on in the background and I'll be using my phone to communicate with whoever I'm communicating with and sometimes I'll have my laptop open for research as well.' (Australia: 7)

I'Il be watching the television and I have the computer, I am like, Internet shopping and I'm watching a show and sometimes I pause it - I don't want to miss something, or I'll rewind it back, because you know, Sky has that feature.' (U.K.: 7)

\section{Creation of media synergies}

The study revealed the creation of media synergies by respondents in the U.K., Germany and Australia, where media are combined based on their specific cognitive requirements. In practice, this is accomplished by choosing specifically chosen compatible combinations of media, which do not conflict in terms of their inherent characteristics. This may be achieved by matching a primarily visual medium with a primarily aural medium. Furthermore, the combination of a visual, aural and a medium which needs only intermittent attention allow three or more media to be used; for example, 'magazine with the mobile and the music' (U.K.: 1); 'I guess those three, mobile phone, music and Internet. They're all things that would be used together' (U.K.:11).

Respondents describe an array of multiple media use situations involving media activities which require only intermittent attention; where for much of the time the user is simply monitoring or waiting for the media to become active (such as social media, text messages and emails). The research shows clear evidence of temporary pauses in multitasking, allowing the two media to be managed more effectively, such as the pausing of a television programme to reply to a text message. 
This finding is particularly interesting, as it reveals that respondents are aware of the negative effects of multitasking and as a result choose to suspend the behaviour at certain times to manage these effects, as illustrated in the following scenarios:

If I'm doing things like social media and watching TV, it will probably be on my iMac together. So, I will have one window open top left which will be the TV, right half of my computer screen will have my social media on there, I'll have web pages open there. Bottom left hand may have my emails that I'll be scrolling through.' (U.K.: 6)

'If you're watching a film and you want to Google something quickly, you'll pause the film... I'd pause the film, look it up and then go back to watching the film.' (U.K.: 11)

\section{Restrictions on multiple media use}

Another management strategy, employed by respondents across our three samples is the use of selfimposed restrictions. Two types of restrictions were noted, media restrictions and topic restrictions. Examples of media restrictions include; switching a mobile 'phone to silent to allow greater control over when it is used, or consciously ignoring one medium, 'I simply turn it off in my brain and ignore $i t^{\prime}$ (Germany: 11).

'When I sometimes watch debates on TV, I consciously put my iPad or iPhone aside and focus on TV. Otherwise I can't follow the content of the discussion. I need to concentrate on one medium then.' (Germany: 8)

'Putting my phone on silent, that's really the only thing that helps me manage it, and sometimes ignoring the emails or ignoring the instant messages or just ignoring. That's really the only two ways, I never turn my phone off, ever' (U.K.: 5)

Respondents also restrict the number of topics accessed when they feel overwhelmed with content. A media multitasking session may start with many topics, but as activities become chaotic and there is a perceived need to focus, respondents limit the topics to a manageable number.

'Sometimes there is no limit. I'll start off with something important and then the next thing I know I'm looking up YouTube videos... and I won't even know how it's come up. But if something needs to be done, then I'll focus on fewer topics so that I'm not too distracted.' (Australia: 8)

\section{Discussion}

\section{Theoretical implications}

This study deepens our understanding of the complexities of the behavioural phenomenon of multiple media use, appreciably extending and updating the work of Bardhi et al. (2010). The 
comprehensive investigation reported in this paper offers a detailed understanding of the multiple media use of thirty four Digital Native respondents in the U.K., Germany and Australia, revealing many more similarities than differences across the three samples. Contributing to a deeper understanding of individuals' approaches to multiple media use; these findings offer enhanced insights into the ways in which switching occurs; between two, three and four media during multitasking. Moreover, in this study, the development of personal 'media multitasking portfolios' is ascertained; in which multiple media combinations are tailored by individuals' to meet the particular needs of a specific setting. This finding provides a notable contribution to understanding of individuals' behaviour in this domain.

Multitasking with combinations of two, three or four media is confirmed as commonplace among our Digital Native respondents. Moreover, multiple media use is confirmed as a routine feature of everyday life, encompassing the use of separate media and single device media multitasking, in accordance with previous empirical work (for example, Pilotta et al., 2004; Pilotta \& Schulz, 2005; Foehr, 2006; Brasel \& Gips, 2011). The practice of stopping and starting media activities or switching between media while multitasking, is also a common feature of this study, affirming the findings of Brasel and Gips, (2011) and Yeykelis et al., (2014). Although contrary to some previous work, which proposes that multiple media consumption occurs simultaneously (for example, Pilotta \& Schultz, 2005), this finding concurs with more recent observational work (Brasel \& Gips, 2011; Yeykelis et al., 2014).

An appraisal of the perceived benefits of media multitasking, reveals broad similarities between respondents in the U.K., Germany and Australia who report; efficiency, assimilation, control and engagement, in accordance with Bardhi et al. (2010). Furthermore, this study exposes the additional perceived benefits of: emotional and social gratification; connectedness and extended information access in relation to their multiple media use. The detection of social gratification and extended 
information concurs with findings by Hwang et al., (2016), while the desire for connectedness accords with connection in previous work by Kononova and Chiang (2015). Similarities are also evident across the three country samples in relation to the cited costs of multiple media use. Perceived costs include: inefficiency; chaos and disengagement, in agreement with Bardhi et al. (2010). The noteworthy additional costs; of an inability to focus and reduction in attention are also revealed in this study. In accordance with Bardhi et al. (2010), paradoxical findings are apparent in relation to this aspect of the findings: for example; efficiency and engagement, cited in all samples as benefits, also emerge as costs (inefficiency and disengagement), signifying tensions for individuals involved in multiple media use.

Extending the work of Bardhi et al. (2010), individuals' strategies for managing media multitasking behaviour identified among our U.K., German and Australian respondents provide a substantial advance in understanding of the complexities of this phenomenon. The creation of: media hierarchies; media synergies and the use of self-imposed restrictions as deliberate approaches to manage multiple media use are revealed by respondents in this study. Media hierarchies are created by multiple media consumers to control the intensity with which they interact with various media. In this setting, respondents' consume some media as foreground and others as background media, confirming earlier findings (Pilotta \& Schultz, 2005; Bardhi et al., 2010; Brasel \& Gips, 2011). Media synergies are achieved by choosing specific compatible media combinations, based on their cognitive requirements, in order that they do not conflict in terms of their inherent characteristics. Additionally, multiple media use combinations involving media activities requiring only intermittent attention, are purposefully selected. For example, media synergy can be achieved by complementing a primarily visual medium with a primarily aural medium, alongside a medium requiring only intermittent attention; such as the combination of searching the internet and listening to radio, while intermittently attending to social media activity. An important finding of this study is that respondents organise their multiple media use through the creation of media hierarchies and media 
synergies to generate personal 'media multitasking portfolios' for a particular media use setting, such as relaxing at home in the evening. Furthermore, to facilitate greater control or concentration, whilst releasing some of the associated tensions associated with multiple media use; respondents' employ self-imposed media and topic restrictions. Examples include; switching a mobile 'phone to silent (or putting it away) and purposely choosing to ignore particular media channels; for example, text messages or social media alerts.

\section{Implications for practitioners}

Consumers' multiple media use augments the complex nature of the selection of media channels within the IMC planning function. Further, in a media multitasking scenario, the use of more than one medium creates a situation in which advertising messages may be avoided, as revealed in this study. Conversely, multiple media use may support potential opportunities for the occurrence of media synergy (Laurie and Mortimer, 2011), if the 'media multitasking portfolios' of target audiences in various settings are sufficiently well understood by media planners, facilitating IMC campaigns. Furthermore, to exploit the advantages associated with media synergy, marketing communications practitioners must endeavour to create campaigns which relate a single story across a multi-media campaign. Scolari (2009) argues that distinctive media content must be delivered through appropriate media channels to form a cohesive narrative, named transmedia storytelling. Using this technique, engagement by an audience with each media event is considered to increase the comprehension, entertainment and affection of the consumer for the story and accordingly for the brand. Consistently, it is probable that the transmedia storytelling technique is inextricably connected with the concept of IMC, in which a single-minded and coherent message is recommended as part of an integrated strategy, to facilitate the consistency of brand image (for example, Hackley, 2010; Percy \& Rosenbaum-Elliott, 2016). Accordingly, to achieve maximum communication benefit and effectiveness on behalf of clients, an enhanced understanding of the 
intricacies of multiple media use, provided by the findings of this study, is valuable to marketing communications and media planners.

\section{Limitations and future research directions}

The limitations associated with qualitative research methods are applicable to this study, based on a non-probability sample of thirty four Digital Natives. Despite the conscientious application of quality criteria and the triangulation achieved by the three country sample, the findings of this study should not be generalised to a wider population of Digital Natives. Nevertheless, these findings provide a substantial contribution to existing work in the domain of multiple media use and are of enormous value to academics and practitioners. Future research plans include corresponding qualitative work among Digital Immigrants (those born before 1980), who learned to use digital technology, rather than growing up with it. As a consequence, 'digital' is their second language (Prensky, 2001) and it is postulated that they may have differing views from Digital Native samples. In addition, future empirical work should further examine the 'media multitasking portfolios' of Digital Natives and Digital Immigrants in diverse settings; for example, at home or on the move, to establish the range of prevalent combinations.

\section{References}

Bardhi, F., Rohm. A.J. \& Sultan, F. (2010) 'Tuning in and tuning out: media multitasking among young consumers.' Journal of Consumer Behaviour, 9, 316-332. doi: 10.1002/cb.320

Brasel, S.A. \& Gips, J. (2011) 'Media Multitasking Behaviour: Concurrent Television and Computer Usage', Cyber Psychology, Behaviour and Social Networking, 14 (9), 527-534. doi: 10.1089/cyber.2010.0350

Bryman, A. \& Bell, E. (2011) Business Research Methods. Oxford: Oxford University Press.

Carrier, L.M., Cheever, N.A., Rosen, L.D. Benitez, S. \& Chang, J. (2009) 'Multitasking across generations: Multitasking choices and difficulty ratings in three generations of Americans', Computers in Human Behaviour, 25, 483-489. doi: 10.1016/j.chb.2008.10.012

Carrier, L.M., Rosen, L. D., Cheever, N.A. \& Lim, A.F. (2015) 'Causes, effects and practicalities of everyday multitasking', Developmental Review, 35, 64-78. doi: 10.1016/j.dr.2014.12.005.0273-2297

Danaher, P. (2007) In: The Sage Handbook of Advertising. Edited by Tellis, G.J. \& Ambler, T., London: Sage. 
Denzin, N.K. and Lincoln, Y.S. (eds) (2011) The Sage Handbook of Qualitative Research (4 ${ }^{\text {th }}$ edn). London: Sage.

Delbridge, K.A. (2000). Individual differences in multitasking ability: Exploring a nomological network. Unpublished Doctoral dissertation, Mitchigan State University, East Lansing.

Duff, B., Yoon, G., Wang, Z. \& Anghelcev, G. (2014) 'Doing It All: An Exploratory Study of Predictors of Media Multitasking', Journal of Interactive Advertising, 14 (1), 11-23. doi:

10.1080/15252019.2014.884480

Enoch, G. \& Johnson, K. (2010) 'Cracking the Cross-Media Code. How to use Single-Source Measures to examine Media Cannibalisation and Convergence', Journal of Advertising Research, June, 125-136. doi: $10.2501 / \mathrm{s} 0021849910091294$

Fill, C. \& Turnbull, S. (2016) Marketing Communications - discovery, creation and conversations. $7^{\text {th }}$ Edition. Harlow: Pearson Education.

Foehr, U.G. (2006) 'Media Multitasking Among American Youth: Prevalence, predictors and pairings'. Menlo Park. CA: Henry Kaiser Family Foundation.

Hackley, C.E. (2010) Advertising and Promotion: An Integrated Marketing Communications Approach. $2^{\text {nd }}$ edn. London: Sage Publications.

Jeong, S-H. \& Fishbein, M. (2007) 'Predictors of Multitasking with Media: Media Factors and Audience Factors', Media Psychology, 10 (3), 364-384. doi: 10.1080/15213260701532948

Keller, K.L. (2001) Mastering the Marketing Communications Mix: Micro and Macro Perspectives on Integrated Marketing Communications Programs, 17:7, 819-847.

Kitchen, P.J. \& Schultz, D.E. (1998) IMC - A UK Ad' Agency Perspective, Journal of Marketing Management, 14:5, 465-485. doi: 10.1362/026725798784867806

Kliatchko, J. (2008) Revisiting the IMC construct: A revised definition and four pillars, International Journal of Advertising, 27(1), 113-160. doi: 10.1080/02650487.2008.11073043

König, C.J. \& Waller, M.J. (2010) 'Time for reflection: A critical examination of polychronicity', Human Performance, 23, 173-190. doi: 10.1080/08959281003621703

Kononova, A. \& Chiang, Y-H (2015) Why do we multitask with media? Predictors of media multitasking among Internet users in the United States and Taiwan. Computers in Human Behaviour, 50, 31-41. doi: 10.1016/jchb.2015.03.052

Laurie, S. \& Mortimer, K. (2011) 'IMC is dead. Long live IMC: Academics versus practitioners' views, Journal of Marketing Management, 27:13-14, 1464-1478. doi: 10.1080/0267257X.2011.627367

Lin, L. (2009). Breadth-Biased versus Focused Cognitive Control in Media Multitasking Behaviours. Proceedings of the National Academy of Sciences of the United States of America, 106, (37) 1552115522. doi: 10.1073/pnas.0908642106

Malhotra, N. \& Birks, D. (2007) Marketing Research: an applied approach, $3^{\text {rd }}$ ed., [online] Pearson Education, Available from: http://www.dawsonera.com [Accessed: 20 March 2013].

Mariampolski, H. (2001) Qualitative Market Research: A Comprehensive Guide, London: Sage. 
Miles, M.B. \& Huberman, A.M. (1994) Qualitative Data Analysis: An expanded Sourcebook, London: Sage.

Percy, L. \& Rosenbaum-Elliot, R. (2016) Strategic Advertising Management. Fifth Edition: Oxford.

Petty, R.E. \& Caccioppo, J.T. (1986) Communication and Persuasion: Central and Peripheral Routes to Attitude Change, New York: Springer.

Pilotta, J.J., Shultz, D., Drenik, G \& Rist, P. (2004) 'Simultaneous media usage: a critical consumer orientation to media planning', Journal of Consumer Behaviour, 3 (3), 285-292. doi: 10.1002/cb.141

Pilotta, J.J. \& Shultz, D. (2005) 'Simultaneous Media Experience and Synesthesia', Journal of Advertising Research, 45 (1), 19-26. doi: 10.1017/s0021849905050087

Poposki, E.M. \& Oswald, F.L. (2010). The Multitasking Preference Inventory: Toward an improved measure of individual differences in polychronicity. Human Performance, 23, 247-264. doi: $10.1080 / 08959285.2010 .487843$

Prensky, M. (2001) 'Digital Natives, Digital Immigrants', On the Horizon, Vol. 9 (5), 1-6 Available from: http://www.marcprensky.com/writing/Prensky\%20\%20Digital\%20Natives,\%20Digital\%20Immigrants\%20-\%20Part1.pdf [Accessed: 26 March 2013].

Reuters Institute (2012) Reuters Institute Digital Report 2012. Available at: https//reutersinstitute.politics.ox.ac.uk/fileadmin/documents/Publications/Other_Publications/Reut ers_Institute_Digital_Report.pdf (Accessed: $9^{\text {th }}$ March 2013)

Rettie, R., Robinson, H., Radke, A. \& Ye, X. (2008) 'CAQDAS: a supplementary tool for qualitative market research', Qualitative Market Research: An International Journal, 11(1), 76-88.

Richie, J. and Lewis, J. (2003) Qualitative Research Practice A guide for Social Science Students and Researchers. London: Sage.

Rosen, L.D., Carrier, L.M. \& Cheever, N.A. (2013) 'Facebook and texting made me do it: Media induced task switching while studying', Computers in Human Behaviour, 29, 948-958. doi: 10.1016/j.chb.2012.12.001

Savin-Baden, M. and Howell Major, C. (2013) Qualitative Research: The essential guide to theory and practice, Abingdon: Routledge.

Scolari, C.A. (2009) 'Transmedia Storytelling: Implicit Consumers, Narrative Worlds and Branding in Contemporary Media Production', International Journal of Communications, 3, 586-606.

Srivastava, J., Nakazawa, M. \& Chen, Y. (2016) 'Online, mixed and offline media multitasking: Role of cultural, socio-demographic, and media factors', Computers in Human Behaviour, 62, 720-729. doi: 10.1016/j.chb.2016.04.0400747-5632

Voorveld, H.A.M. (2011). Media multitasking and the effectiveness of combining online and radio advertising, Computers in Human Behaviour, 27, 2200-2206. doi: 10.1016/jchb.2011.06.016

Wang, Z., David, P., Srivastava, J., Powers, S., Brady, C., D’Angelo, J. \& Moreland, J. (2012) 'Behavioural Performance and visual attention in communication multitasking: A comparison between instant messaging and online voice chat', Computers in Human Behaviour, 28, 968-975. doi: 10.1016/jchb.2011.12.018 
Webster, J.G. \& Ksiazek, T.B. (2012) 'The Dynamics of Audience Fragmentation: Public Attention in an Age of Digital Media', Journal of Communication, 62 (3), 493-593. doi: 10.1111/j.1460-

2466.2011.01616.x

Yardley, L. (2000) 'Dilemmas in Qualitative Health Research', Psychology and Health, 15, 215-28.

Yeykelis, L., Cummings, J.J. \& Reeves, B. (2014) 'Multitasking on a single device: Arousal and the Frequency, Anticipation, and Prediction of Switching Between Media Content on a Computer', Journal of Communication, 64, 167-192. doi: 10.1111/jcom.12070 